\title{
PONTRYAGIN DUALITY FOR TOPOLOGICAL MODULES
}

\author{
JOE FLOOD
}

\begin{abstract}
A Pontryagin duality for topological modules over any locally compact ring is described, using the Pontryagin dual of the ring as character module.
\end{abstract}

Several authors have attempted to extend the Pontryagin duality for locally compact Abelian groups to classes of topological modules over topological rings. G. C. Preston [6] obtained duality for modules over the $p$-adic integers and fields and their local products, M. F. Smith [7] for linear spaces, and M. D. Levin [4] for modules over discrete commutative rings. In each case, the character module, or module representing the contravariant dual functor, was taken to be the Pontryagin dual of the ring $R$, which was shown to be canonically an $R$-module.

It is proven that duality holds for locally compact modules over locally compact rings, using the dual $\hat{R}$ of $R$ as character module. A duality is valuable, in that it increases the possibilities for structural dismemberment of objects, and enhances the functional richness of the category under consideration. Parts of the result have been hinted at in the folklore of topological rings, where Kaplansky [3] and others have elegantly used characters to obtain structure theorems. A few obvious applications are included.

1. Suppose $X$ and $Y$ are objects in Top, the category of topological spaces and continuous functions. Denote by $(X, Y)$ the space of continuous functions from $X$ to $Y$ given the compact-open topology. Then a subbasis for this topology is given by the sets $U(K, O)=\{f \mid f(K) \subset O\}$, where $K$ is any compact subset of $X$ and $O$ a member of any subbasis for the topology of $Y$. The map $X \times Y \rightarrow(X, Y)$ is the action on objects of a functor from Top ${ }^{\text {op }} \times$ Top to Top [5, p. 181].

A well-known property of the compact-open topology gives a 1-1 correspondence between continuous functions $A \rightarrow(X, Y)$ and functions from $A \times X$ to $Y$ continuous on sets of the form $A \times K$, where $K$ is a locally compact subset of $X$. Another property is the following:

2. Lemma. Suppose $\psi: X \times Y \rightarrow Z$ is in Top, and $A$ is a topological space. The natural mappings

(i) ${ }_{A} \psi: X \times(A, Y) \rightarrow(A, Z)$, where ${ }_{A} \psi(x, f)(a)=\psi(x, f(a))$ and

Received by the editors May 10, 1978 and, in revised form, November 1, 1978.

AMS (MOS) subject classifications (1970). Primary 22D35, $13 \mathrm{~J} 99$. 
(ii) $\psi_{A}: X \times(Z, A) \rightarrow(Y, A)$, where $\psi_{A}(x, g)(y)=g \circ \psi(x, y)$, for $X$ locally compact

are continuous.

Proof. (i) Suppose, for $x_{0}$ in $X$ and $f_{0}$ in $(A, Y)$, that ${ }_{A} \psi\left(x_{0}, f_{0}\right)$ is in subbasic $U(K, O) \subset(A, Z)$, where $K \subset A$ is compact and $O \subset Z$ is open. Then $\psi\left(x_{0}, f_{0}(K)\right) \subset O$. Choose neighbourhoods $V$ of $x_{0}$ and $W$ of $f_{0}(K)$ such that $\psi(V \times W) \subset O$, using the compactness of $f_{0}(K)$. Then ${ }_{A} \psi(V, U(K, W)) \subset U(K, O)$, and ${ }_{A} \psi$ is continuous; since the inverse image of each subbasic set is a neighbourhood of each of its points.

(ii) For $x$ in $X$ and $g$ in $(Z, A)$, suppose $g \circ \psi(x, K) \subset O$, for $O \subset A$ open and $K \subset Y$ compact. If $V$ is a compact neighbourhood of $x$, then $K^{\prime}=\psi(V$ $\times K)$ is compact, and

$$
\psi_{A}\left(V, U\left(K^{\prime}, O\right)\right) \subset U(K, O)
$$

for $V$ small enough, so $\psi_{A}$ is continuous.

It is assumed in the following that a topological ring is a ring with identity and a group topology making the ring multiplication jointly continuous. If $R$ is a topological ring and $M, N$ are two topological left (right) $R$-modules, so that the module multiplication is jointly continuous, let ${ }_{R}[M, N]\left([M, N]_{R}\right)$ be the space of continuous $R$-morphisms between $M$ and $N$ equipped with the compact-open topology. If $R$ is the integers, so that $M$ and $N$ are topological Abelian groups, use the shorthand $[M, N]$ for the space of continuous group morphisms.

3. Proposition. If $G$ is a topological Abelian group and $M$ a topological left $R$-module, then

(a) $[G, M]$ is a topological left $R$-module,

(b) $[M, G]$ is a topological right $R$-module if $R$ is locally compact.

Proof. Certainly $[M, G]$ and $[G, M]$ are topological Abelian groups under pointwise addition.

If $\mathbf{m : ~} R \times M \rightarrow M$ is the continuous module multiplication, it is standard $[4$, p. 51] that the restrictions of the composition maps of Lemma 2,

${ }_{G} \mathbf{m}: R \times[G, M] \rightarrow[G, M]$ and $\mathbf{m}_{G}:[M, G] \times R \rightarrow[M, G]$

make $[M, G]$ and $[G, M]$ into left and right $R$-modules. Since by Lemma 2 these functions are continuous, both modules are topological.

This result holds also with the words "right" and "left" interchanged.

So if TAb is the category of topological Abelian groups and continuous morphisms, and ${ }_{R} \mathrm{TAb}\left(\mathrm{TAb}_{R}\right)$ the category of topological left (right) $R$ modules and continuous morphisms, the bifunctor $[-,-]$ (defined on objects as above, and if $f: G_{2} \rightarrow G_{1}, g: H_{1} \rightarrow H_{2}$ are in TAb, $[f, g]:\left[G_{1}, H_{1}\right] \rightarrow$ $\left[G_{2}, H_{2}\right]$ takes $\alpha$ in $\left[G_{1}, H_{1}\right]$ to $\left.g \circ \alpha \circ f\right)$, takes

$$
\mathrm{TAb}^{\mathrm{op}} \times{ }_{R} \mathrm{TAb} \rightarrow{ }_{R} \mathrm{TAb} \text { and }{ }_{R} \mathrm{TAb}^{\mathrm{op}} \times \mathrm{TAb} \rightarrow \mathrm{TAb}_{R} .
$$


And if $M$ is in ${ }_{R} \mathrm{TAb}$ and $G$ is taken to be the toroidal group $\mathrm{T}$, the Pontryagin dual $\hat{M}=[M, T]$ is in $\mathrm{TAb}_{R}$. This particular case was stated without proof by Goldman and Sah [1], who found interesting structural applications.

4. Bimodules. Suppose $N$ is a topological $R-R$ bimodule. That is, $N$ is both a topological left and right $R$ module satisfying $(r n) s=r(n s)$ for $r, s$ in $R$ and $n$ in $N$. Then if $M$ is in ${ }_{R}$ TAb, ${ }_{R}[M, N]$ is a right submodule of $[M, N]$ with the right structure determined by $N$, since for $r, s$ in $R, m$ in $M$ and $f$ in ${ }_{R}[M, N]$,

$$
f s(r m)=f(r m) s=r(f(m) s)=r(f s)(m) .
$$

A contravariant functor from ${ }_{R} \mathrm{TAb}$ to $\mathrm{TAb}_{R}$ is defined by ${ }_{R}[-, N]$. Any functor naturally equivalent to a functor of this type is said to be represented by the bimodule $N$.

If $G$ is any Abelian topological group, $[R, G]$ is a bimodule by Proposition 3(b), since $R$ is, for locally compact $R$. There is canonical counit morphism $\eta$ : $[R, G] \rightarrow G$ in $\mathrm{TAb}$, evaluating each function at the identity of $R$.

5. Proposition. If $\boldsymbol{R}$ is a locally compact topological ring and $\boldsymbol{G}$ a topological Abelian group, the contravariant functor $[-, G]$ on ${ }_{R} \mathrm{TAb}$ may be represented by the bimodule $[R, G]$.

Proof. If $\eta:[R, G] \rightarrow G$ is the continuous morphism evaluating a function at the identity, then if $M$ is a topological left $R$-module, the continuous morphism (natural in $M$ )

$$
[M,[R, G]] \stackrel{\left[1_{M}, \eta\right]}{\rightarrow}[M, G]
$$

has as inverse on the submodule ${ }_{R}[M,[R, G]]$ of $R$-morphisms the map $\mu$ : $f \rightarrow \hat{f}$, where for $f$ in $[M, G], m$ in $M$ and $r$ in $R, \hat{f}(m)(r)=f(r m)$.

To show $\mu$ continuous, if $U\left(K_{1}, U\left(K_{2}, O\right)\right)$ is a subbasic open set in ${ }_{R}[M$, $[R, G]$, with $K_{1} \subset M$ and $K_{2} \subset R$ compact, and $O$ open $\subset G$, its inverse image is $U\left(\mathrm{~m}\left(K_{1} \times K_{2}\right), O\right)$ in $[M, G]$, and since by continuity of the multiplication $\mathrm{m}$, the set $\mathrm{m}\left(K_{1} \times K_{2}\right) \subset M$ is compact, the inverse image is open in $[M, G]$.

We have shown, incidentally, that the functor $[R,-]$ taking $\mathrm{TAb}$ to ${ }_{R} \mathrm{TAb}$ is a right adjoint in a strong sense to the forgetful functor.

EXAMPLE. If $G$ is the toroidal group $\mathbf{T}$, and $\mathbf{R}$ is the reals, with $\hat{\mathbf{R}}$ identified with $\mathbf{R}$ under the mapping $r \rightarrow e^{2 \pi i r x}[2,23.27 \mathrm{e}], \eta: \mathbf{R} \rightarrow \mathbf{T}$ may be taken to be the exponential mapping $x \rightarrow e^{2 \pi i x}$. Then every continuous character of a topological linear space can be obtained from a continuous linear functional by composition with $\eta[2,23.32]$.

Taking $G=\mathrm{T}$ in Proposition 5 , so that $[M, G]=\hat{M}$, we obtain: 
6. THEOREM. If $R$ is a locally compact ring and $M$ a topological left $R$-module, the Pontryagin dual $\hat{M}$ is isomorphic to ${ }_{R}[M, \hat{R}]$. If $M$ satisfies Pontryagin duality as a topological group (e.g. if $M$ is locally compact Hausdorff) then $M$ is isomorphic to $\left.{ }_{R}[M, \hat{R}], \hat{R}\right]_{R}$ under the unit embedding $e_{R}$ : $m \rightarrow \hat{m}$, where $\hat{m}(\alpha)=\alpha(m)$ for $m$ in $M$ and $\alpha$ in ${ }_{R}[M, \hat{R}]$.

Proof. It remains to show that the composite isomorphism

$$
M \stackrel{e}{\rightarrow} \hat{\hat{M}^{\prime}} \stackrel{\mu_{M}}{\rightarrow}[\hat{M}, \hat{R}]_{R} \stackrel{\left.\left[1_{M}, \eta\right], 1_{\dot{R}}\right]_{R}}{\rightarrow}\left[{ }_{R}[M, \hat{R}], \hat{R}\right]_{R}
$$

where $e(m)(f)=f(m)$ for $f$ in $\hat{M}$ and $m$ in $M$, and $\mu,[1, \eta]$ are the natural isomorphisms of Proposition 5, is equal to $e_{R}$. The composite takes $m$ to $\bar{m}$, in $\left.{ }_{R}[M, \hat{R}], \hat{R}\right]_{R}$, where for $r \in R, \alpha \in{ }_{R}[M, \hat{R}]$,

$$
\bar{m}(\alpha)(r)=\alpha(r m)(1)=\alpha(m)(r), \text { so } \bar{m}=e_{R}(m)
$$

and if $e$ is an isomorphism, so is $e_{R}$.

7. Corollary. The categories of locally compact Hausdorff left and right modules over a locally compact ring are dual under the Pontryagin functor.

AppLications. In what follows, all rings and modules are locally compact Hausdorff.

8. Proposition [1]. A ring with no open left ideals has no compact right modules.

Proof. If $R$ has no open left ideals it has no discrete left modules and hence no compact right modules.

If $N$ is a closed submodule of module $M$, then $N_{\perp}=\{f \in \hat{M}: f(N)=0\}$ is a closed submodule of $\hat{M}$, and $\hat{M} / N_{\perp}$ is isomorphic to $\hat{N}[2,24.11]$. Also $N_{\perp}$ is isomorphic to the dual of the quotient module $M / N[2,23.25]$.

9. Proposition. Every neighbourhood of the identity in a compact $R$-module $M$ contains a closed module $N$ such that $M / N$ is isomorphic to a compact subgroup of a finite product of copies of the dual $\hat{R}$.

Proof. If $V$ is a neighbourhood of the identity in $M$, by duality $V$ contains some $U(F, W)$, where $F$ is a finite subset with $n$ elements of the discrete module $\hat{M}$, and $W \subset T$ is open. If $P$ is the submodule generated by $F$, there is a continuous map $\phi: R^{n} \rightarrow P$. Taking $N=P_{\perp} \subset V$, we have

$$
M / N \cong \hat{P} \stackrel{\hat{\phi}}{\rightarrow}\left(\widehat{R^{n}}\right) \cong\left(\widehat{R^{n}}\right) \quad[2,23.18] .
$$

Since $\phi$ is surjective and $\hat{P}$ is compact, $\hat{\phi}$ is an isomorphism into.

A module is profinite if it is a projective limit of finite modules.

10. Proposition. A left module over a compact ring is profinite if and only if it is compact.

Proof. Profinite modules are compact, as closed submodules of products of finite modules. 
If $M$ is a compact module over compact $R$, the discrete module $\hat{M}$ is the colimit (direct limit) of its finitely generated submodules, all of which are finite (they are discrete and compact). Since the dual functor is selfadjoint on the right, it takes colimits to limits, so that $M$ is a (projective) limit of finite modules, since the dual of a finite module is finite.

\section{REFERENCES}

1. O. Goldman and C. H. Sah, Locally compact rings of special type, J. Algebra 11 (1969), 363-454.

2. E. Hewitt and K. A. Ross, Abstract harmonic analysis. I, Springer-Verlag, Berlin, 1963.

3. I. Kaplansky, Locally compact rings, Amer. J. Math. 70 (1948), 447-459.

4. M. D. Levin, Locally compact modules, J. Algebra 24 (1973), 25-53.

5. S. Mac Lane, Categories for the working mathematician, Graduate Texts in Math., vol. 5, Springer-Verlag, Berlin, 1971.

6. G. C. Preston, On locally compact totally disconnected Abelian groups and their character groups, Pacific J. Math. 6 (1956), 121-134.

7. M. F. Smith, The Pontrjagin duality theorem in linear spaces, Ann. of Math. (2) 56 (1952), 248-253.

Current address: Division of BuIlding Research, CSIRO, P. O. Box 56, Highett, Victoria 3190, Australia 